\title{
Capacitive Near-Field Communication for Ubiquitous Interaction and Perception
}

\author{
Tobias Grosse-Puppendahl ${ }^{1}$, Sebastian Herber ${ }^{1}$, Raphael Wimmer ${ }^{2}$, Frank Englert ${ }^{3}$, \\ Sebastian Beck ${ }^{1}$, Julian von Wilmsdorff ${ }^{1}$, Reiner Wichert ${ }^{1}$, and Arjan Kuijper ${ }^{1,3}$ \\ ${ }^{1}$ Fraunhofer IGD, Fraunhoferstr. 5, 64283 Darmstadt, Germany, \{firstname.lastname $\}$ igd.fraunhofer.de \\ ${ }^{2}$ University of Regensburg, Universitätsstraße 31, 93053 Regensburg, Germany, raphael.wimmer@ur.de \\ ${ }^{3}$ Technische Universität Darmstadt, Karolinenplatz 5, 64289 Darmstadt, Germany \\ frank.englert@kom.tu-darmstadt.de, arjan.kuijper@gris.tu-darmstadt.de
}

\begin{abstract}
Smart objects within instrumented environments offer an always available and intuitive way of interacting with a system. Connecting these objects to other objects in range or even to smartphones and computers, enables substantially innovative interaction and sensing approaches. In this paper, we investigate the concept of Capacitive Near-Field Communication to enable ubiquitous interaction with everyday objects in a short-range spatial context. Our central contribution is a generic framework describing and evaluating this communication method in Ubiquitous Computing. We prove the relevance of our approach by an open-source implementation of a low-cost object tag and a transceiver offering a high-quality communication link at typical distances up to $15 \mathrm{~cm}$. Moreover, we present three case studies considering tangible interaction for the visually impaired, natural interaction with everyday objects, and sleeping behavior analysis.
\end{abstract}

\section{Author Keywords}

capacitive sensing; capacitive near-field communication; capacitive communication; intrabody communication

\section{ACM Classification Keywords}

H.5.2. Information Interfaces and Presentation: User Interfaces - Graphical user interfaces; Input devices \& strategies

\section{INTRODUCTION}

Recent advances in interaction technology have introduced a paradigm shift from traditional interaction methods, like graphical user interfaces, to ubiquitous interaction with a multitude of cooperating objects [16]. Here, manipulations of objects or their perception of the environment influence the system's state - which allows for both implicit and explicit interaction. A key requirement for ubiquitous interaction is the communication between objects among each other and the external computing infrastructure.

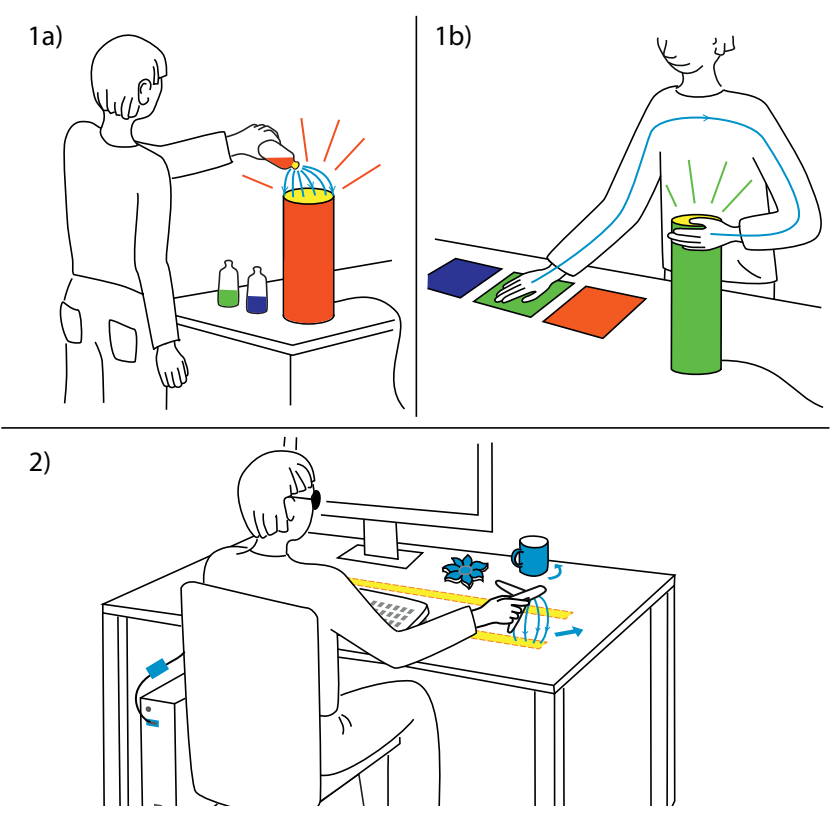

Figure 1. CapNFC use-cases: (1a/b) Interacting naturally with everyday objects by transmitting object-related information through air or the human body, (2) using everyday objects to support blind users in interacting with a computer.

When choosing a communication method, developers have to accept certain trade-offs which include cost, form factors, and power consumption. This challenge becomes even more relevant when considering a high number of interactive objects, which are supposed to be small, have heterogeneous form factors, run on a very limited power supply, and are required to be low-cost. Technologies like RFID or NFC represent a very suitable alternative to medium-range communication, especially when the information is exchanged in a short-range spatial context. Moving from the inductive to the capacitive domain, a human's conductive properties allow for indirectly incorporating the perception of body parts, touches and proximity into the communication technique. Especially in ubiquitous computing, this property leverages its full potential as a means for short-range communications using the air or the human body as a communication channel [39]. 
In this paper we present and discuss Capacitive Near-Field Communication - CapNFC - as an ultra low-power, touchor proximity-mediated alternative to existing wireless communication techniques. Existing smart objects can be easily adapted to use CapNFC as only a single microcontroller output pin is required for unidirectional communication. In terms of form factors, CapNFC is extremely flexible, supporting the integration into beds as well as small objects, such as body-worn sensors. CapNFC employs capacitive coupling between nearby conductive electrodes to transmit information over distances of up to $15 \mathrm{~cm}$ through air. Furthermore, the technology allows for communicating messages through the human body or measuring the proximity to human body parts. It utilizes frequencies of $1 \mathrm{kHz}$ to $1 \mathrm{Mhz}$, with wavelengths that are a multitude larger than the transmitting and receiving electrodes. Therefore, CapNFC acts in the quasi-electrostatic regime, eliminating the necessity for high-frequency design and complex hardware components. This results in a very small displacement current flowing from a transmitter to a receiver electrode, enabling ultra-low power operation.

Capacitive coupling as a communication method has found interest in intrabody communications [39], information exchange using touchscreens [35, 38] or access schemes in proximity sensing $[28,40]$. Instead of focussing on a specific approach, we propose a generalized methodology for Capacitive Near-Field Communication in UbiComp. Therefore, we offer the following contributions in our paper:

1. We introduce and evaluate a novel generic framework for Capacitive Near-Field Communications in ubiquitous environments. We identify a set of operating modes and the corresponding interaction techniques.

2. Three case studies support the applicability of CapNFC in Ubiquitous Computing: (i) natural interaction paradigms with everyday devices, (ii) smart objects assisting blind users in interacting with their computer, and (iii) a smart bed with wearable sensors for sleeping behavior analysis. The first two case studies are partly depicted in Figure 1.

3. We describe, compare and evaluate the concepts of CapNFC in sufficient detail to allow others to re-implement it. Open-source schematics and source code enables the community to quickly implement CapNFC applications.

\section{GENERIC FRAMEWORK FOR CAPACITIVE NEAR-FIELD COMMUNICATION}

CapNFC relies on capacitive coupling between a transmitter and a receiver electrode [40]. Applying an alternating voltage to one electrode and connecting the second electrode to ground causes a changing electric field between both electrodes. With CapNFC, the induced displacement current is measured on the receiver side. The energy-saving potential in ubiquitous communications can be regarded by physical considerations. CapNFC acts in the quasi-electrostatic regime and thus without any wave-propagation at its electrodes. This property solely enables a very small displacement current to flow from the transmitting electrode to the environment. In order to communicate by means of capacitive coupling, voltages oscillating at low frequencies $(\leq 1 \mathrm{MHz})$ are applied to the transmitter electrode. The receiver amplifies the induced displacement current and decodes the message. Various encoding schemes may be used on top of this physical communication channel. Communication range and robustness depend strongly on the size of the transmitter electrodes and their voltages levels. Higher voltages increase the displacement current and lead to a better performance. Bigger electrodes allow for better capacitive coupling [13] but also pick up more background noise. In our examples, electrode sizes vary from $1 \mathrm{~cm}^{2}$ to $100 \mathrm{~cm}^{2}$.

In this work, we apply the concept of CapNFC to ubiquitous interaction with everyday objects. Each object which is touched or manipulated by a user establishes an information flow and communicates its state to all objects within range. Devices with extended interaction capabilities, such as smartphones and computers, can interpret the information and generate a response to object manipulations in their environment. In the following, we discuss general operating modes, describe our reference implementation comprising hardware and communication protocol, present considerations for electrode placement, and document performance and energy consumption of our prototypes.

\section{Operating modes}

All communication techniques based on capacitive coupling do not only require a transmitter and receiver electrode, but also a connection to a common ground. Only when two objects share a common ground, a displacement current is able to flow from one electrode to the other. A common ground may be established by sharing a ground between both circuits or by connecting each circuit to the environment's ground. However, as only very small displacement currents flow back and forth, CapNFC also works if a transmitter and a receiver are grounded via weak capacitive coupling to the environment's ground. This connection can be generated by a user touching the object, or a table on which the object is placed.

Many ubiquitous artifacts and devices are portable and must thus be battery-powered. Therefore, they lack a shared ground or direct connection to the ground, which is a prerequisite for communication. Nevertheless, humans usually have good capacitive coupling to the environment's ground [39]. Thus, establishing a common ground between two objects can be achieved by embedding ground electrodes into the casing of an object and connecting the electrode to the battery's negative terminal. When a human touches the ground electrode or is in close proximity to it, capacitive coupling between the ground electrode and the environment's ground is sufficiently strong to allow a displacement current to flow. A very strong coupling to the environment's ground, for example by directly connecting the objects to a grounded power supply, enables objects to communicate messages through the human body [39]. Based on these properties, we identified three different operating modes for CapNFC that allow for reliable data communication in ubiquitous environments, as depicted in Figure 2. The CapNFC framework embraces and utilizes the need for a common ground connection between sender and receiver by making it a central part of the supported interaction methods. 


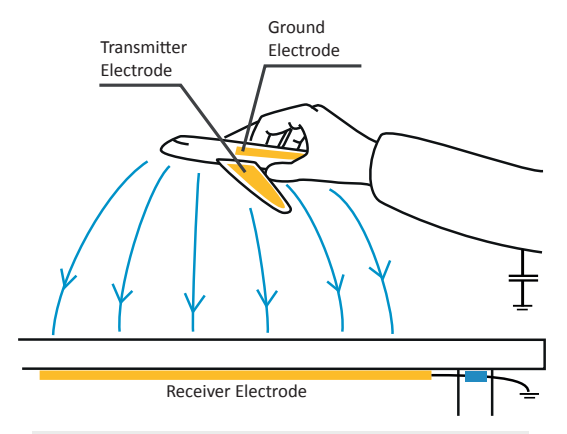

Ground-Coupling by Touch

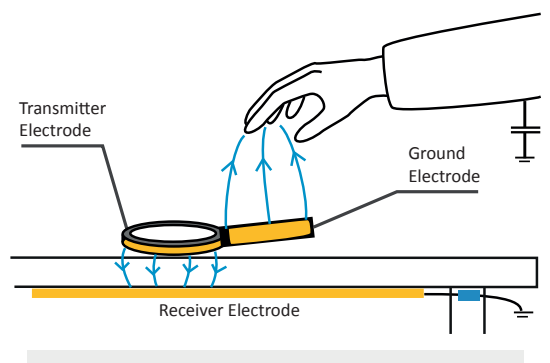

Ground-Coupling by Proximity

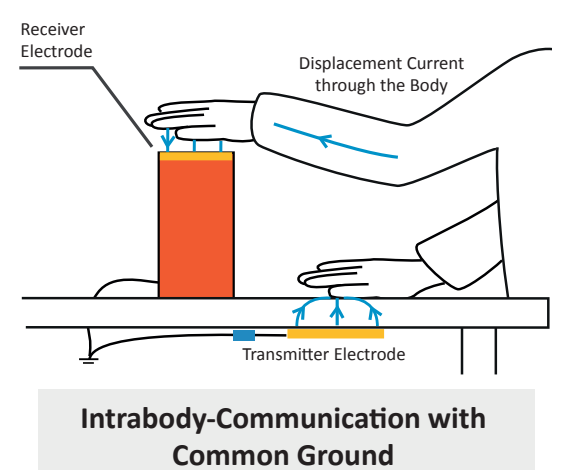

Common Ground

Figure 2. CapNFC offers three operating modes that depend on the ground-coupling of objects: Ground-coupling by touch for indirect touch recognition, ground-coupling by proximity for sensing a person approaching an object, and sharing a common ground for intrabody-communication.

\section{Ground-Coupling by Touch}

In this mode, the receiver object is connected to the environment's ground, and the transmitter is a battery-powered object. Besides the transmit electrode located in the smart object, a small additional electrode is connected to the battery's negative power supply. Whenever the user is in very close proximity to this electrode or touches it, the weak capacitive coupling to the grounded human body enables an information flow from the transmitter to the receiver. Therefore, the ground electrode indirectly acts as capacitive touch sensor.

\section{Ground-Coupling by Proximity}

In this operating mode, a receiver is connected to the environment's ground, and a battery-powered smart object is located in close proximity to the receiver. In addition to the transmitter electrode, the battery-powered object has a builtin ground electrode. When the user approaches this electrode with a body part the capacitive coupling to the environment's ground increases. By measuring the signal strength in the receiver, it is possible to recognize an approaching human hand in distances up to $15 \mathrm{~cm}$.

\section{Intrabody-Communication with a Common Ground}

When both objects are directly connected via a common ground, an information flow can be established through the user's body [39]. This enables the user to touch two objects and thus establish communication between both of them. In CapNFC, this mode is primarily applicable for stationary installed objects, as they may be directly connected to a grounded power supply.

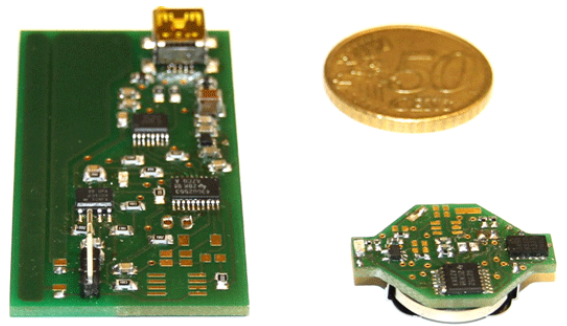

Figure 3. Two different CapNFC components: A tag (right) with an embedded accelerometer and a transceiver (left). The hardware is only partly equipped to allow for experimenting with active sending filters.

\section{Reference Implementation}

The CapNFC concept can be adapted for a multitude of applications. However, we believe that ubiquitous user interfaces benefit the most from CapNFC. Before designing the CapNFC reference hardware and software, we defined constraints that must be fulfilled in order to allow for a high number of low-cost smart objects: (1) transmitting information should not require any additional active hardware components (crystals, amplifiers, ...) except for a single microcontroller; (2) receiving information must consume a low amount of processing power and energy; (3) the method has to be suitable for low-power microcontrollers that run on frequencies as low as $1 \mathrm{MHz}$. For Ubiquitous Computing, we prioritize these goals over a high data rate.

Based on these design-centered decisions, we implemented two hardware components: a transceiver and a low-power transmitter tag, as shown in Figure 3. The transceiver comprises a microcontroller (MSP430G2553), a serial-toUSB converter (FT230XS), an operational amplifier IC (OPA2344), and a voltage regulator (TPS79733). It can be connected to a PC or smart phone via USB. The tag contains only a low-power microcontroller (MSP430G2352) and an accelerometer (ADXL345). It is powered by a 3 Volt coin cell monitored by a voltage regulator (TPS79730). The tag can be attached to an object and is only able to transmit predefined and context-dependent messages via capacitive coupling, using all three modes identified in the previous section. The accelerometer allows saving power and supporting gestural interaction with a tagged object. It activates CapNFC communication whenever the tag is moved or a pre-defined gesture is detected.

\section{Communication Method and Protocol}

Figure 4 shows an exemplary CapNFC communication setup. The communication principle itself is based on broadcasting messages; the range around an object can be represented by a spatial context on which messages of predefined types are published. Components may also publish higher level information composed of previously received as well as data measured by themselves.

Due to the design constraints and the limited communication space, we decided not to include carrier-sense multiple access 


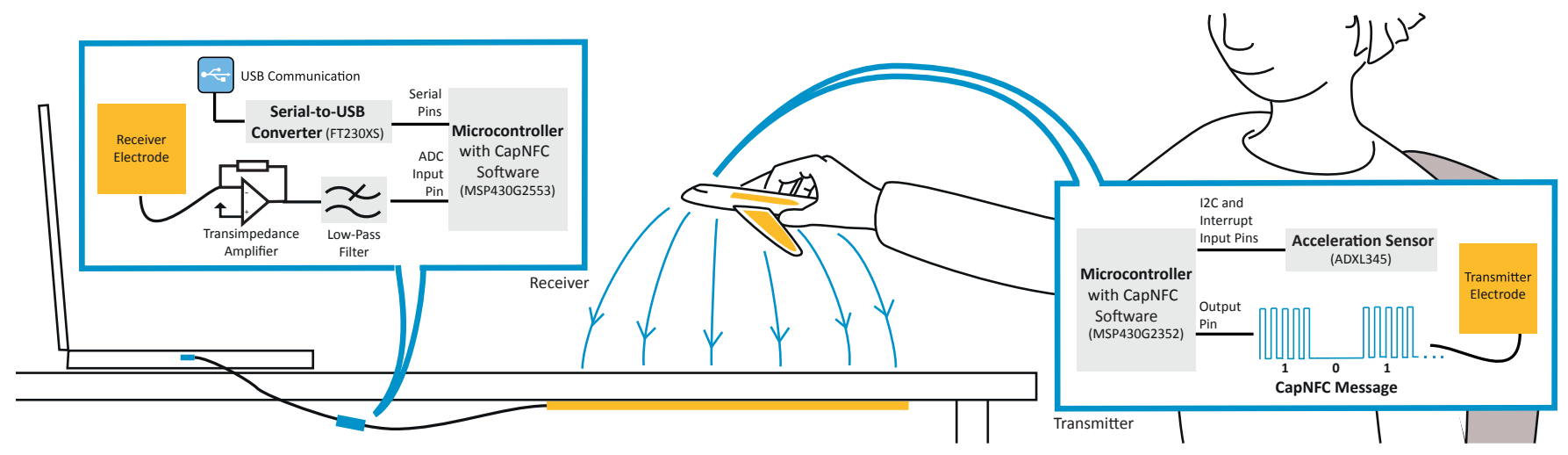

Figure 4. An exemplary CapNFC setup: A toy airplane communicates with a computer by transmitting its movement related data using a quasielectrostatic field. The airplane is equipped with a low-power CapNFC tag, consuming $280 \mu A$ in total while transmitting a message. The receiver amplifies the induced displacement current, decodes the messages and hands them to the computer via USB.

techniques (CSMA) to avoid collisions when multiple transmitters access the communication medium. Instead, the existence of data transmission collisions is accepted deliberately in our protocol. This fact enables smart objects that solely employ a low-cost microcontroller without additional hardware. These objects are only capable of transmitting messages, without being able to receive any information from other objects. Due to this simple concept, existing smart objects can be easily extended to support CapNFC communication as only a single additional output pin at a microcontroller is needed.

Information is transmitted via a $3.0-3.3 \mathrm{~V}$ rectangular-shaped signal, oscillating at a frequency of $10 \mathrm{kHz}$. The signal can be easily created using a microcontroller's pulse-widthmodulation module, which is a common functionality even in low-cost hardware. In order to represent binary information, the signal is switched on and off (On-Off-Keying), allowing for a data rate of $2 \mathrm{KBit} / \mathrm{s}$. As illustrated in Figure 5, a message begins with two high bits which represent a start condition, followed by a synchronization byte of succeeding 1-0 transitions (0xAA), the length of the message (1 byte), the message type ( 1 byte), a sender address and a succeeding payload (less than 30 bytes including parity bytes). The reason for the payload length limitation lies in potential clockdrift among the different hardware components. The receiver of a message synchronizes with each 1-0 and 0-1 transition. However, when the number of transitions is low, the clockdrift would be too high for payloads longer than 30 bytes.

\begin{tabular}{|c|c|c|c|c|c|c|}
\hline $\begin{array}{l}\text { Start } \\
10 \text { Bit }\end{array}$ & $\begin{array}{l}\text { Data Length } \\
8 \text { Bit }\end{array}$ & $\begin{array}{l}\text { Type } \\
8 \text { Bit }\end{array}$ & $\begin{array}{l}\text { Sender } \\
8 \text { Bit }\end{array}$ & & $\begin{array}{c}\text { Data } \\
0-20 \text { Bytes }\end{array}$ & \\
\hline Sync & 6 Byte & $\begin{array}{l}\text { Inertial } \\
\text { Sensor }\end{array}$ & $\begin{array}{l}\text { Sender Address of } \\
\text { Magnifier Glass }\end{array}$ & $\begin{array}{c}\text { Acceleration } \\
\text { x-axis } \\
\text { (2 Byte) }\end{array}$ & $\begin{array}{c}\text { Acceleration } \\
y \text {-axis } \\
\text { (2 Byte) }\end{array}$ & $\begin{array}{c}\text { Acceleration } \\
\text { z-axis } \\
\text { (2 Byte) }\end{array}$ \\
\hline $\begin{array}{l}0 \times 03 \\
0 \times A A\end{array}$ & 6 & $0 \times 01$ & $0 \times 12$ & 10 & -15 & 140 \\
\hline Sync & 3 Byte & Color Picker & $\begin{array}{l}\text { Sender Address of } \\
\text { Mobile Phone }\end{array}$ & $\begin{array}{c}\text { Red } \\
\text { (1 Byte) }\end{array}$ & $\begin{array}{l}\text { Green } \\
\text { (1 Byte) }\end{array}$ & $\begin{array}{l}\text { Blue } \\
\text { (1 Byte) }\end{array}$ \\
\hline $\begin{array}{l}0 \times 03 \\
\text { OxAA }\end{array}$ & 3 & $0 \times 20$ & $0 \times 20$ & 255 & 50 & 50 \\
\hline
\end{tabular}

Figure 5. Our CapNFC communication protocol. The message format can be seen on top, two exemplary messages below. All messages are secured with parity bytes for bit error correction.
In order to receive data, which is optional in CapNFC, a receiver employs a transimpedance amplifier followed by a low-pass filter [28]. It requires three simple hardware components - a low-cost operational amplifier, two resistors and a capacitor. The receiver's microcontroller samples the signal at $40 \mathrm{kHz}$, and reconstructs the transmitted information with the Goertzel algorithm [12]. This computationally inexpensive algorithm is used to detect the presence of the transmitted signal oscillating at $10 \mathrm{kHz}$. In the following, parity bytes are used to restore potentially corrupted bits and an optional CRC checksum ensures the integrity of a message.

\section{Electrode Placements}

As stated in the introduction of CapNFC operating modes, communication between two objects requires a weak capacitive coupling to a common ground. Besides connecting the object directly to a common ground, a weak ground coupling can be set up by a human touching or approaching a ground electrode. In order to establish a suitable coupling to the environment, this electrode is attached to the object's negative supply voltage and placed near the object's surface or directly connected to conductive parts of the object. In his work about Personal Area Networks, Zimmerman determined a capacitance of $110 \mathrm{pF}$ [39] for a current flowing from an isolated human body to the environment's ground.

If the object is only supposed to communicate while being touched or when a hand is in proximity, it is necessary to place a small electrode somewhere near the regions the hand approaches. The bigger the ground electrode gets, the better a displacement current is able to flow from the transmitter electrode to a different object's receiver electrode. Transmitting information without physical touch can be realized by placing an electrode close to other objects having an indirect connection to the environment's common ground, for example a table's wooden surface. The ground electrodes may also be isolated or located within the object as it is only necessary that alternating current is able to flow. In the easiest scenario, the transmitting or receiving electrodes of a CapNFC-enabled object are the object's conductive body itself. However, electrodes can be constituted of different materials, for example conductive threads, aluminum foil, transparent ITO lay- 

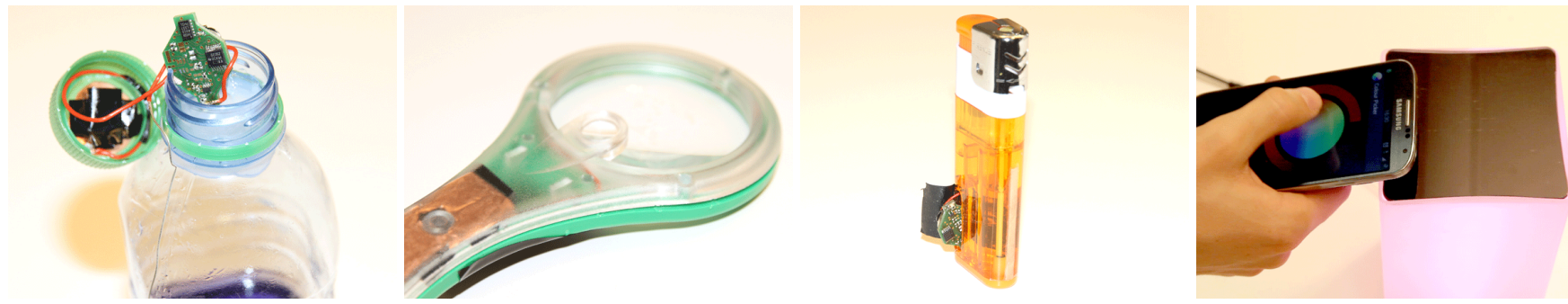

Figure 6. Different electrode placements: (1) The transmitter electrode is placed directly under the bottle cap, (2) the magnifying glass has a big ground electrode in the region where the user touches the object, the transmitter electrode is placed around the glass, (3) the lighter uses its conductive part as a transmitter electrode, and (4) the mobile phone's transmitter electrode is placed at its back, the lamp's receiver electrode is under the black surface.

ers or inkjet-printed silver [17]. The low surface conductivity of transparent or printed materials themselves decreases the performance of capacitive coupling applications only slightly [13]. This fact can also be transferred to capacitive communications and enables a designer to choose from a wide variety of materials with different properties. Figure 6 shows possible electrode locations and placements of some exemplary CapNFC components.

\section{Performance Evaluation}

We evaluated the performance of the presented communication method by assessing our reference implementation with different tagged objects having uni- and bidirectional communication abilities. The experiments were carried out in a standard living environment, with the objects being placed on a wooden desk. As a performance indicator, the signal-tonoise ratio $\left(\mathrm{SNR}=10 \log _{10}\left(P_{\text {signal }} / P_{\text {noise }}\right)\right)$ was applied. In the following, we present evaluation results for all three CapNFC operating modes.

Figure 7 depicts the SNR for different transmitter/receiver distances employing ground-coupling by touch. Therefore, we combined two copper electrodes of different sizes and cross-evaluated the SNR. Interestingly, the evaluation revealed that one should rather use larger transmitter electrodes, than enlarging the size of the receiver electrode. Enlarging the receiver electrode's size will result in a greater amount of noise, whereas the strength of the received signal does not increase proportionally. This is a negative property for ubiquitous interaction, as it is very difficult to increase the trans-

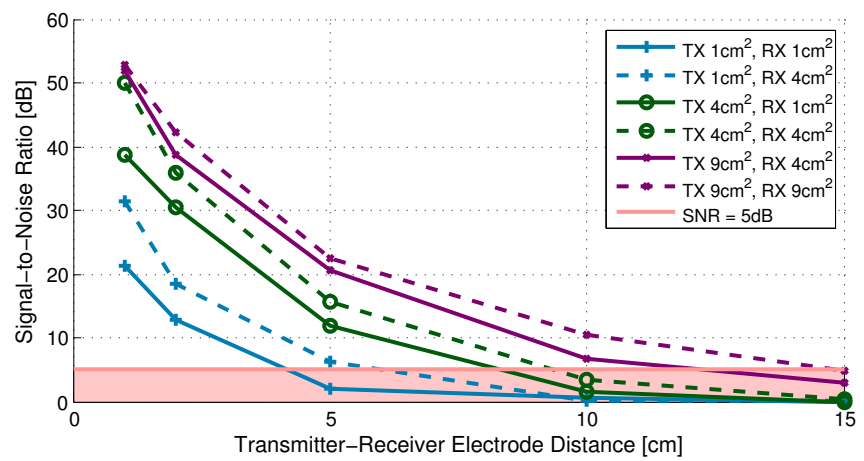

Figure 7. Ground-Coupling by Touch: The Signal-To-Noise Ratio with different receiver/transmitter distances and electrode sizes. The $5 \mathrm{~dB}$ SNR threshold results in a bit error rate (BER) of more than $20 \%$, which means that many messages are corrupted. mitter electrode's size in small battery-powered objects. Enlarging the receiver electrode's size, which may be located under the desk, is usually easier as the space is not as constrained. Depending on the electrodes' sizes, messages can be transmitted in hand distances up to $15 \mathrm{~cm}$.

Figure 8 depicts the evaluation results for ground-coupling by proximity. In this experiment, we use a battery-powered tag with differently sized ground and transmitter electrodes, located in close distance to a receiver with a corresponding large receiver electrode. A human hand was used to approach the ground electrode at different distances with multiple repetitions of the experiment. The experiment shows that the detection distance increases with larger ground electrodes. The evaluation of larger ground electrodes revealed that the weak coupling to the environment's ground, for example the table, is sufficient for transmitting messages. In these cases, the SNR remains constant even after removing the hand. Alas, inferring a certain distance from a given SNR requires strong initial assumptions about the object's location and orientation. Nevertheless, differences in the SNR between succeeding messages can be exploited to detect hand presence and proximity differences.

The evaluation results for intrabody with common ground are depicted in Figure 9. We evaluated different touch types for coupling a transmitter and a receiver electrode. Therefore, we isolated each electrode under a wooden surface and touched it with one, two, three fingers, and the whole hand. Surprisingly to us, the SNR was not affected very strongly when touching

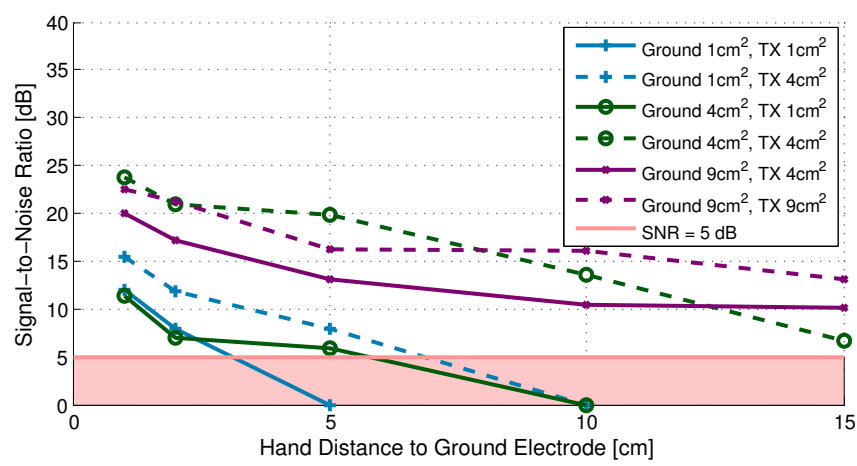

Figure 8. Ground-Coupling by Proximity: The object is placed in little distance to the receiver electrode. The diagram shows the SNR for different ground and transmitter electrode sizes. By approaching the hand to the ground electrode, the SNR increases. 


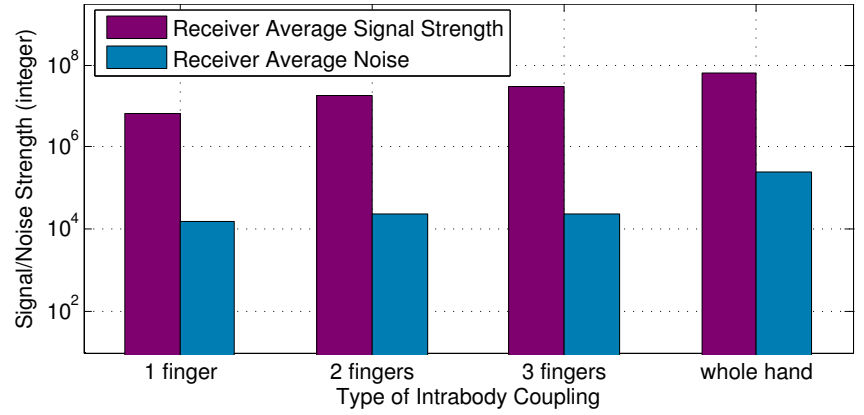

Figure 9. Intrabody with Common Ground: The signal/noise strength is given as an integer value, representing the average result after computing a window with the Goertzel algorithm.

the electrodes with more fingers. However, when analyzing the signal and the noise levels separately from each other, the signal as well as the noise levels increase when the coupling is made through a larger area, e.g. a hand. This interesting property is very useful for gesture or grasp recognition, for example by remotely detecting when a finger was added or removed from an object.

Figure 10 shows the relation between SNR, bit error rate (BER) and packet error rate (PER) in our reference implementation. The PER does not only depend on the BER after error correction, but also on the detection of the message preamble, which is currently not fault tolerant. When using larger receiver electrodes, the already high noise variance increases and leads to a decreased BER and PER. Possible reasons can be seen in noise generated by devices operating at similar frequencies, e.g. switch-mode power supplies.

In the following, we discuss the energy consumption of CapNFC and our reference implementation. The energy required for succeedingly loading and unloading the capacitance between transmitter electrode and grounded parts has only little influence on the implementation's energy consumption. This fact is very important, as it only adds little energy consumption to existing systems while leveraging communication abilities based on capacitive coupling. Moreover, in the future this property allows for creating tags powered by energy-harvesting or capacitive power transfer [20], very sim-

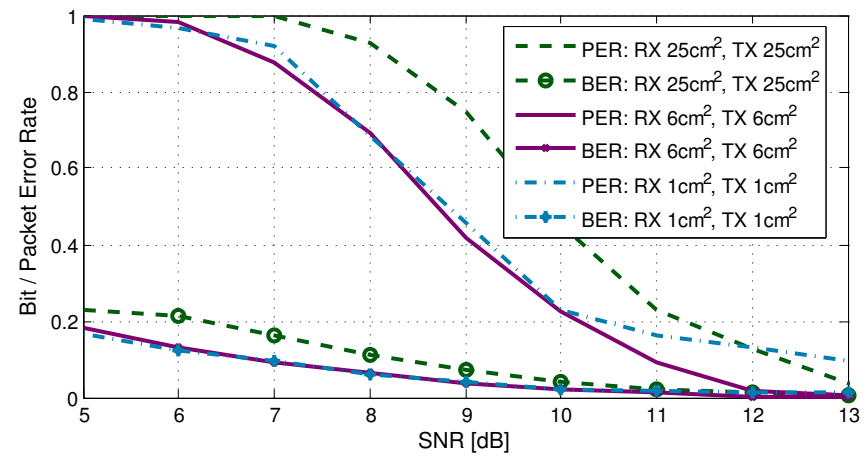

Figure 10. The relation between SNR, the bit and packet error rate in the region from $5-13 \mathrm{~dB}$. The packets had a data length of 3 byte $(+1$ byte sync) secured with 3 parity bytes $((8,4)$-Hamming). ilar to RFID. Basically, only a very simple low-power microcontroller is necessary to transmit data. For example, while transmitting 10 inertial samples per second, our low power tag consumes less than $88 \mu A$ at $3.0 \mathrm{~V}$ on average. Receiving messages requires an additional operational amplifier, which consumes $750 \mu \mathrm{A}$ in our implementation and a more powerful microcontroller for signal processing. However, the energy consumption of a receiver can be reduced further by choosing a different amplifier. Currently, our amplifier supports a very high slew rate to experiment with significantly higher frequencies than $10 \mathrm{kHz}$. In terms of energy consumption, it can be concluded that CapNFC supports extremely low-power implementations, especially suitable for the usage in smart, energy-constrained, objects. A comparison of CapNFC with other technologies, such as RFID, will be given in the next section.

\section{RELATED WORK AND COMPETING TECHNOLOGIES}

\section{Capacitive Communications and Sensing}

Using capacitive coupling for information exchange is applied in a wide variety of application scenarios. Amongst others, it is used for communication between objects and multitouch enabled devices $[35,38]$, and communication via the human body [39]. Prototyping capacitive sensing applications has made significant advances in the last years due to inkjet-based printing [7, 17] or low-cost foil cutters [26].

\section{Capacitive Coupling through Air and Direct Contact}

Some prototypes of tangible objects allow sending short messages to a device with a capacitive touchscreen by simulating touch events $[35,38]$. The presented communication technique works with arbitrary capacitive touchscreens and requires only simple hardware for the sender that is integrated into a finger ring. Obviously, the communication is unidirectional from the sender to the multi-touch device and has a low bandwidth limited by the scanning frequency of the touchscreen. In the domain of indoor user localization, Valtonen et al. use conductive tiles on which the human body acts as a transmitter electrode [34]. A receiver placed on the ceiling or within a wall identifies the tiles a user steps on. Cohn et al. presented a gesture recognition system based on electric fields produced by devices within the user's home environment $[5,6]$. In contrast to the methods discussed before, the human body acts as a receiver electrode while a wearable hardware component analyzes the received signal.

\section{Intrabody Communication}

Capacitive coupling can also enable two devices to exchange information using the human body as a communication medium $[11,39]$. This principle was firstly investigated by Zimmerman for realizing Personal Area Networks [39]. In his work, he points out that all communicating nodes require not only a capacitive coupling to the human body but also a coupling to a common ground potential. Since then, this principle was picked up by many researchers, for example to identify different users at a touch screen [8]. Park et al. proposed the Touch and Play (TAP) system [23] for creating a link between media storage devices and output devices through the human body. In a concrete application scenario, a photo was transferred from a digital camera to a printer. 


\section{Proximity and Grasp Sensing using Capacitive Coupling}

In the domain of proximity sensing, capacitive coupling can be used for recognizing gestures $[14,28]$, sensing user locations [29] or their activities [13, 37]. Also, capacitive sensing was used in wearable devices to measure muscle contractions for activity recognition [4]. The approaches rarely transmit information, they rather use traditional capacitive sensing techniques. However, modulation approaches like codedivision multiple access or frequency-division multiple access can also be found in this domain $[13,28]$. In order to discern different ways a user touches an object, capacitive sensing technology such as swept-frequency capacitive sensing $[15,25]$ or time-domain reflectometry [36] were employed.

\section{Competing Communication Technologies Long-Range Wireless Communication}

The most important difference between CapNFC and longrange wireless communications can be seen in the supported transmission range and energy consumption. Technologies like Bluetooth or ZigBee support transmission ranges of 1 $100 \mathrm{~m}$, whereas CapNFC usually operates in a range up to $15 \mathrm{~cm}$. Compared to wireless communications, CapNFC is easier and cheaper to implement, and consumes significantly less energy while transmitting and receiving data. On the other hand, using wireless communications offers several advantages like a full-duplex communication stack, greater distances and higher data rates. Therefore, the desired communication range, constraints in energy consumption and communication abilities are the most important decision factors.

\section{Inductive RFID / NFC}

In contrast to Capacitive NFC, Inductive NFC (RFID) has found many applications due to its commercial breakthrough and available passive tags. RFID was used in smart environments for interacting with everyday objects, for example coupling a mobile phone to a stereo device [3] or enabling an information flow between touch-enabled surfaces [10]. Moreover, it is used as a bridging technology for establishing a confidential pairing with other wireless communication services and for mobile micropayment solutions [9]. In the following, we compare the power consumption of our CapNFC implementation to an ultra-low power RFID (NFC) implementation given in [32]. Prior to this discussion it must be noted that both implementations have very different levels of technological maturity and RFID's communication concept provides more elaborate features [21]. RFID relies on a reader-central infrastructure supporting passive tags, whereas CapNFC's communication mechanism is based on broadcasting and a decentralized infrastructure. Also, the data rates strongly differ between both exemplary implementations (CapNFC: 2kBit/s; RFID/NFC: 424kBit/s) [32].

In order to compare the two approaches, we apply a simplified measure of energy consumption per bit $E_{b t}=$ $\frac{\text { Energy Consumption }}{\text { Maximum Data Rate }}$ When transmitting, our CapNFC implementation's energy consumption is lower $(360 \mathrm{nW} / \mathrm{Bit})$ than the given RFID implementation (825 nWs/Bit) [33]. While the RFID reader requires more than twice the energy per bit, it is also able to power passive tags, which CapNFC does not do. A comparison of both communication channels, i.e. ignoring the peripherals' energy consumptions, shows a larger difference. An output energy of $235 \mathrm{nWs}$ /Bit is typical for RFID $[31,33]$. CapNFC's output energy varies from $0.225 \mathrm{nWs} / \mathrm{Bit}$ while transmitting through air to $2.475 \mathrm{nWs} / \mathrm{Bit}$ while transmitting through the human body. This suggests that more mature CapNFC implementations can lead to an energy consumption which is many magnitudes smaller than RFID. The main reason is the small amount of energy needed to charge the capacitance between transmit electrode and environment. CapNFC's energy consumption while receiving information is mainly induced by the transimpedance amplifier and the signal processing on the microcontroller. As we did not optimize the transceiver board for low-power operation, signal processing leads to an energy consumption of $7.5 \mu \mathrm{Ws} / \mathrm{Bit}$. Nevertheless, we expect a great potential for reducing energy consumption in the receiver part.

Due to CapNFC's early development stage, only few approaches have been made to create passively powered communication devices. However, power transmission using capacitive coupling, as employed in [20], proves the potential of passively powered capacitive sensor tags. Even though the comparison of energy consumption between both technologies is very difficult, especially the low energy consumption for transmitting information features CapNFC for use in ultra-low power devices with unidirectional communication abilities. We also see potentials in the combination of energy harvesting techniques [22] to realize passive tags.

RFID does not require a common ground between communicating nodes which is desirable for many applications However, the need for establishing a capacitive coupling to a common ground in CapNFC enables numerous opportunities for embedding interactive properties in an interaction system, as described in the technical framework section. The different CapNFC operating modes allow for intrabody communication, proximity awareness and indirect touch sensing. Comparing CapNFC's electrode placements to the placements of RFID antennas, one can achieve similar form factors, as described in the technical evaluation and the following case studies. The need for a common ground in CapNFC requires an additional ground electrode, raising the complexity of tagging an object. A very interesting property in CapNFC is the equipment of large objects with communication abilities, which would require multiple RFID readers. For example, a bed can be equipped with simple conductive wires to build a large receiver area. In conclusion, the decision of using CapNFC or RFID strongly depends on the application scenario, an overview of our discussion is outlined in Figure 12.

\begin{tabular}{|c|c|}
\hline Inductive RFID / NFC & CapNFC \\
\hline$+\quad$ Tagging of objects is & Flexibili \\
\hline $\begin{aligned} & \text { very } \\
-\quad & \text { Larg }\end{aligned}$ & $\begin{array}{r}\text { 1ntr } \\
+\quad \text { Ver }\end{array}$ \\
\hline $\begin{array}{ll} & \text { easy to tag } \\
\text { - } \quad \text { Limited interaction }\end{array}$ & $\begin{array}{r}\text { us } \\
+\quad \operatorname{Pr}\end{array}$ \\
\hline $\begin{array}{r}\text { mod } \\
+\quad \text { Pass }\end{array}$ & ion \\
\hline $\begin{array}{l}\text { - High peak currents during } \\
\text { communication }\end{array}$ & $\begin{array}{l}+\quad \begin{array}{l}\text { Very energy-efficient information } \\
\text { exchange }\end{array}\end{array}$ \\
\hline$+\quad$ No ground-coupling is required & At least two electrodes needed \\
\hline
\end{tabular}

Figure 12. Comparison of RFID with CapNFC properties for UbiComp. 

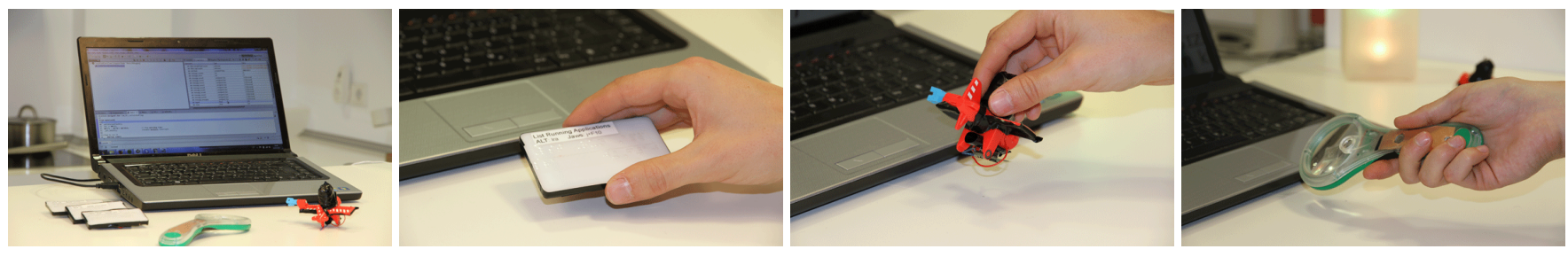

Figure 11. An exemplary workplace equipped with CapNFC-enabled objects for symbolic access to a very specific set of computer functionality. The transceiver is connected to the $\mathrm{PC}$, having a receiver electrode placed under the user's desk.

\section{CASE STUDIES: CAPNFC IN UBIQUITOUS COMPUTING}

Sensing object manipulations in combination with Capacitive Near-Field Communication offers an elegant solution for perceiving a user's environment as well as interesting ubiquitous interaction opportunities. CapNFC has a number of useful properties that are highly desirable in Ubiquitous Computing:

1. Instantaneous, infrastructure-free communication: Using CapNFC, interaction is independent from a server-based infrastructure or central access points. Instead, a multitude of devices within range communicates instantaneously based on a stateless communication protocol.

2. Natural interaction: A user can directly identify and combine the objects required for interaction. By employing additional sensors within an object, the knowledge of object manipulations and movements can be exploited and published in a short-range context. Combining the different CapNFC operating modes can be utilized for enriching the interaction, for example by detecting touch, proximity or communicating messages through the human body.

3. Low energy consumption: Capacitive Near-Field Communication requires significantly less energy than highfrequency wireless communication.

4. Low hardware requirements: Transmitting information is as easy as toggling a pin on a microcontroller, without requiring any additional integrated circuits. Moreover, the layout of transmit and receive electrodes is very flexible in terms of materials, shapes and sizes.

In the following, we present three case studies that benefit from CapNFC and combine the different operating modes in a useful way. In the first use-case, we present a real-world example where blind users interact with a PC using tangible objects. The second use-case is a conceptual study of natural interaction with an everyday object. Thirdly, we present an activity recognition system for sleeping analysis.

\section{Cast Study 1: Tangible Interaction for the Blind}

In this case study, we present a prototypical implementation of a tangible interaction system for visually impaired users. Especially for this target group, tangible objects provide an easy and intuitive way of symbolically accessing computer functions $[19,24]$. To develop a feasible and ergonomic solution, we work in close cooperation with a company specialized in developing products for the blind.

In order to use a computer efficiently, blind people are obliged to learn a high number of keyboard shortcuts and commands that are used to automate the computer. Symbolic access methods that link tangible objects to computer functions can help those people to execute a very specific set of actions, such as opening the weather report or reading their emails. Especially when considering the usage scenario of controlling continuous system properties, such as the reading speed of a screen reader, the usage of tangible objects employing CapNFC enables a very fast and natural way of interaction. Figure 11 shows different tangible objects that were realized for this use-case. A transceiver is connected to a PC and the receiver electrode is deployed under the desk. The first object we realized is a plastic card with braille text. Each card represents a command that is carried out by the computer, for example 'list running applications'. Therefore, when the card is being touched (ground-coupling by touch), the card's ID and its acceleration data can be sent to the computer. When the card is successfully identified, a double tap on the card executes the corresponding command. The second object is a toy airplane used for regulating the reading speed of the screen reader. Leaning the plane forward and backward increases and decreases the speed. The third object - a magnifying glass - operates in two modes: touch and proximity. When the user tries to find the magnifying glass on the desk, the computer is able to play a sound when the hand moves over it. When the glass is touched, it can be leaned in two directions to control the position of the screen magnifier. By employing the signal-to-noise ratio at the receiver, the magnification level can be increased and decreased depending on the glass's distance.

As the behavior of each object is very specific, we implemented context-sensitive audio hints for each object by shaking it. Moreover, acoustic notifications indicate when an object was successfully recognized or when the object is put away. Our interviews and initial experiments with three blind users revealed that the setup must be chosen very carefully, according to the target person or group. As the initial learning process of keyboard-based systems is very difficult, elderly, children, and persons with multiple disabilities represent a suitable target group for the system. People who are solely visually impaired and have no difficulties in learning are probably not going to benefit from the system, as a high productivity can only be achieved when using keyboard shortcuts. Nevertheless, in working life, computer workplaces for blind persons are often equipped with highly specific hardware, in which certain tangible objects could complement traditional interaction methods. The long battery life and very low cost of CapNFC-enabled tangible objects represent very important factors for the acceptance of the tangible interaction system. 


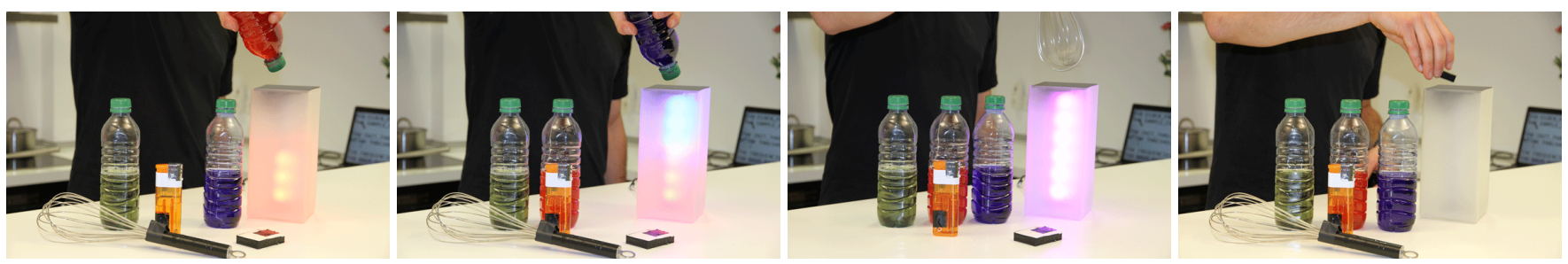

Figure 13. An exemplary workflow for interacting with a smart lamp: The bottles are used to virtually fill up the lamp with the corresponding color. Moving the whisk above the lamp will mix the colors, whereas a gesture with the rubber gum switches off the lamp.

Besides the interviews, we evaluated different objects involved in the case study to demonstrate the suitability of CapNFC communication. Therefore, we observed the SNR as well as the bit-error-rate before data correction (BER = $\frac{\# \text { incorrect bits }}{\text { total } \# \text { of bits }}$. Our measurement results are listed in Figure 14, showing that communication for ground-coupling by touch is very reliable for object distances of $5 \mathrm{~cm}$. As both evaluated transmitting objects have rather small electrodes reaching from $1 \mathrm{~cm}^{2}$ to $2 \mathrm{~cm}^{2}$, the SNR drops to less than $3 \mathrm{~dB}$ at distances above $8 \mathrm{~cm}$. Ground-coupling by proximity easily allows for recognizing when a hand approaches the magnifying glass. Therefore, the object's ground electrode was chosen to be $3 \mathrm{~cm}^{2}$. As depicted in the table, it enables a developer to reliably detect an approaching hand at distances of $5 \mathrm{~cm}$. Due to the bigger ground electrode, the magnifying glass is sufficiently grounded by the table to retain communication abilities without human presence.

\section{Case Study 2: Interaction with Everyday Objects}

As computing and communication technologies advance, more and more household devices and tools gain 'intelligence' and extended configuration possibilities. For example, lamps with adjustable light color and/or time-dependent lighting patterns have been commercially available for some time. However, such advanced features also require more powerful user interfaces in order to leverage their capabilities. Many users usually suffer from poorly designed interfaces, which induced us to transfer a natural interaction technique on a conceptual lighting control.

Figure 13 depicts a way users may naturally interact with the lamp. Therefore, different objects were equipped with CapNFC tags, whereas the lamp employs a transceiver. We stored CapNFC tags in the bottle caps of three bottles filled with differently colored water. When the user moves a bottle cap into the proximity of the lamp, the tag's acceleration values can

\begin{tabular}{|l|l|l|l|l|}
\hline $\begin{array}{l}\text { Operating } \\
\text { Mode }\end{array}$ & Receiver & Sender & $\begin{array}{l}\text { SNR } \\
{[\mathbf{d B}]}\end{array}$ & BER \\
\hline $\begin{array}{l}\text { Ground- } \\
\text { coupling by } \\
\text { touch }\end{array}$ & $\begin{array}{l}\text { Laptop } \\
\text { (electrode in } \\
\text { the desk) }\end{array}$ & $\begin{array}{l}\text { Braille Card } \\
\text { (object distance } 5 \mathrm{~cm} \text { ) }\end{array}$ & 10.47 & $2.50 \%$ \\
\cline { 3 - 5 } & $\begin{array}{l}\text { Airplane } \\
\text { (object distance } 5 \mathrm{~cm} \text { ) }\end{array}$ & 11.40 & $1.40 \%$ \\
\hline \multirow{2}{*}{$\begin{array}{l}\text { Ground- } \\
\text { coupling by } \\
\text { proximity }\end{array}$} & $\begin{array}{l}\text { Laptop } \\
\text { (electrode in } \\
\text { the desk) }\end{array}$ & $\begin{array}{l}\text { Magnifying Glass } \\
\text { (hand distance 1 cm) }\end{array}$ & 8.56 & $4.17 \%$ \\
\cline { 3 - 5 } & $\begin{array}{l}\text { Magnifying Glass } \\
\text { (hand distance 2 cm) }\end{array}$ & 7.93 & $4.39 \%$ \\
\cline { 2 - 5 } & $\begin{array}{l}\text { Magnifying Glass } \\
\text { (hand distance 5 cm) }\end{array}$ & 6.82 & $7.40 \%$ \\
\hline
\end{tabular}

Figure 14. Case study 1 - signal-to-noise ratio and bit error rate evaluation for different tangible objects above a table. be received by the lamp. When the bottle is leaned in the way someone would do to pour out water, the lamp starts filling up in the color of the bottle's contents. The ground-coupling is achieved by a small conductive silver wire, which is touched by the user's hand. A rubber is used to erase the lamp's content, making use of the internal accelerometer. It also features ground-coupling by proximity when being placed on the lamp's surface. In this case, approaching the rubber in distances up to $10 \mathrm{~cm}$ turns the lamp either on and off. Moreover, objects like a lighter or a whale are able to trigger specific lighting profiles like fire or ocean animations. We also placed three transmit electrodes, representing red, green and yellow, on the table. The three tags were connected to the common ground, enabling them to transmit messages through intrabody communication. As soon as the user touches both the lamp and the colored region, the lamp will fill up with the selected color. Using a mobile phone with a transceiver on its back allows the user to directly transmit color values to the lamp using a color picker. In this setup, the device is automatically grounded when touching its metal case.

Figure 15 shows an evaluation of the smart objects involved in the case study. Despite the small transmitting electrode, the lighter showed a very good performance enabling communications in distances up to $10 \mathrm{~cm}$. Here, the lighter's conductive cap was used as a transmit electrode, which even works when the flame is enabled. In the future, we plan to exploit the increasing signal level when using intrabody communication to adjust the lamp's brightness by adding and removing fingers on its surface. The individual signal levels make it difficult to classify such gestures, as the signal has varying offsets influenced by skin conductivity and differences in the contact area. To conclude, this case study underlines the possibility of implementing new interaction paradigms with CapNFC by combining arbitrary objects to realize a natural way of interacting with everyday objects.

\begin{tabular}{|l|l|l|l|l|}
\hline $\begin{array}{l}\text { Operating } \\
\text { Mode }\end{array}$ & Receiver & Sender & $\begin{array}{l}\text { SNR } \\
{[\mathbf{d B}]}\end{array}$ & BER \\
\hline $\begin{array}{l}\text { Ground- } \\
\text { coupling by } \\
\text { touch }\end{array}$ & \multirow{2}{*}{ Lamp } & $\begin{array}{l}\text { Bottle } \\
\text { (object distance 5 cm) }\end{array}$ & 9.36 & $4.14 \%$ \\
\cline { 3 - 5 } & $\begin{array}{l}\text { Lighter } \\
\text { (object distance 5 cm) }\end{array}$ & 10.06 & $1.91 \%$ \\
\hline $\begin{array}{l}\text { Intrabody } \\
\text { with } \\
\text { common } \\
\text { ground }\end{array}$ & Lamp & $\begin{array}{l}\text { Colored Area } \\
\text { (coupling with 1 finger) }\end{array}$ & 9.69 & $2.33 \%$ \\
\cline { 3 - 5 } & $\begin{array}{l}\text { Colored Area } \\
\text { (coupling with 2 fingers) }\end{array}$ & 9.92 & $2.95 \%$ \\
\cline { 2 - 5 } & $\begin{array}{l}\text { Colored Area } \\
\text { (coupling with 1 hand) }\end{array}$ & 10.50 & $1.60 \%$ \\
\hline
\end{tabular}

Figure 15. Case study 2 - evaluation of communication properties for interacting with everyday objects. 

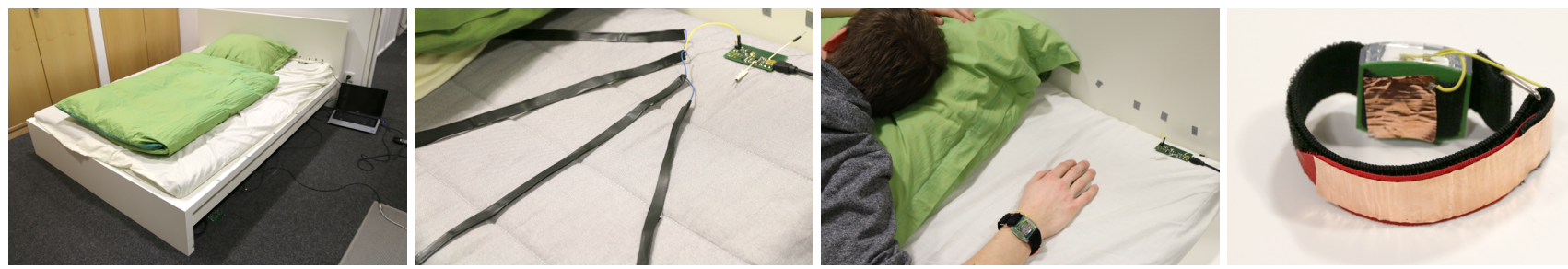

Figure 16. A bed that is able to receive messages from multiple body-worn sensors, for example a wrist-worn accelerometer. The accelerometer is grounded using ground-coupling by touch (inner electrode) and transmits its sensor values to the bed (outer electrode).

\section{Case Study 3: Activity Recognition and Wearables}

Activity recognition in combination with wearable and stationary sensors has been an emerging research topic for the past decade. Various approaches led to a wide variety of sensor-based approaches, for example to recognize activities of daily living $[1,18,30]$ or studying various types of sleeping behavior $[2,27]$.

Transmitting information from multiple sensors to a smart bed can be used for online activity recognition or transferring sensor data collected during the day. Communication distances from the human body to a bedcover are very short, and are therefore well-suited for using CapNFC. In order to demonstrate CapNFC's potentials for sleep recognition, we built a smart bed in combination with a wearable sensor as depicted in Figure 15. The bed incorporates a transceiver with long electrodes placed under the bedcover. The wrist-worn accelerometer periodically reports its sensor data to the bed. It applies ground-coupling by touch with ground electrodes placed near the user's skin and a transmit electrode placed on the wristband's outer part. Furthermore, different regions in the center area near the thigh were equipped with stationary electrodes, transmitting information through intrabody communication when the person lies on them for identifying active regions. This arrangement exemplifies one of CapNFC's strengths: The supported flexible electrode layouts allow for easily equipping large objects with communicational abilities. Due to the different operating modes it is possible to join both, stationary and wearable sensing.

Figure 16 shows the properties of the communication link between the bed, the wristband, and the stationary electrodes. We evaluated two electrode materials within the bed, multiple conductive threads and tiny copper bands placed underneath the bed sheet. Despite the bigger area covered by the

\begin{tabular}{|l|l|l|l|l|}
\hline $\begin{array}{l}\text { Operating } \\
\text { Mode }\end{array}$ & Receiver & Sender & $\begin{array}{l}\text { SNR } \\
{[\mathbf{d B}]}\end{array}$ & BER \\
\hline $\begin{array}{l}\text { Ground- } \\
\text { coupling by } \\
\text { touch }\end{array}$ & Bed (copper) & $\begin{array}{l}\text { Wristband } \\
\text { (object distance 1 cm) }\end{array}$ & 20.31 & $1.12 \%$ \\
\cline { 3 - 5 } & $\begin{array}{l}\text { Wristband } \\
\text { (object distance 8 cm) }\end{array}$ & 11.79 & $1.38 \%$ \\
\hline $\begin{array}{l}\text { Ground- } \\
\text { coupling by } \\
\text { touch }\end{array}$ & Bed (thread) & $\begin{array}{l}\text { Wristband } \\
\text { (object distance 1 cm) }\end{array}$ & 17.65 & $1.52 \%$ \\
\cline { 3 - 5 } & $\begin{array}{l}\text { Wristband } \\
\text { (object distance 8 cm) }\end{array}$ & 11.85 & $1.84 \%$ \\
\hline $\begin{array}{l}\text { Intrabody with } \\
\text { common } \\
\text { ground }\end{array}$ & Bed (copper) & $\begin{array}{l}\text { Stationary transmitter } \\
\text { (copper) }\end{array}$ & 16.67 & $1.61 \%$ \\
\hline
\end{tabular}

Figure 17. Case study 3 - communication performance for stationary and wearable objects communicating with a smart bed. copper bands and their outstanding conductivity, the performance is very similar to using conductive threads. Conductive threads provide a lot of advantages in this case, they are very flexible and can be integrated easily into different kinds of fabric. The evaluation also shows that it is feasible to combine body-worn and stationary appliances to measure bedding postures and fine-grained physical parameters. Nevertheless, when considering wearable devices the data link may always be interrupted by posture changes. Also, using many highly active devices leads to a high number of message collisions.

\section{CONCLUSION \& FUTURE WORK}

As shown in our case studies, CapNFC has proved to be a very suitable technology for ubiquitous interaction and perception. Using CapNFC, it is possible to bridge the gap between a high number of smart objects, low cost, low power consumption and highly interactive system designs. Therefore, the technology represents a suitable companion to RFID with many exciting benefits in interaction design. The implementation is still in a very early stage, heading towards many types of possible applications. A combination of wearable and stationary sensors, as described in the last case study, represents one of the most interesting aspects for future research.

Due to the currently high noise variance, high SNRs are required to obtain a good packet error rate. Besides lowering error rates, more noise-resilient communication methods can also extend the interaction range to $30 \mathrm{~cm}$. In particular, we plan to apply frequencies greater than $100 \mathrm{KHz}$ and use synchronous undersampling to restore the signal [28]. Increasing the carrier frequency will make the signal less prone to noise produced by other electronic devices. Moreover, we will investigate more sophisticated modulation methods like spread-spectrum modulation to avoid problems with narrowband noise [28]. We currently also work on significantly smaller tags and transceivers which consume less power.

Our central contribution in this work is the identification of a CapNFC framework with three operating modes based on different types of ground-coupling. It enables smart objects to communicate in distances of $15 \mathrm{~cm}$ through air, measuring the proximity to human body parts in distances up to $15 \mathrm{~cm}$, and exchanging information through the human body. We discussed the properties of CapNFC, evaluated them in a quantitative manner and presented three case studies proving the suitability of CapNFC in Ubiquitous Computing. Moreover, we support others to implement their own applications by providing source-code, schematics and materials at http://www.opencapsense.org/capnfc. 


\section{REFERENCES}

1. Amft, O., Junker, H., and Tröster, G. Detection of eating and drinking arm gestures using inertial body-worn sensors. In ISWC '05, IEEE (2005), 160-163.

2. Borazio, M., and Van Laerhoven, K. Combining wearable and environmental sensing into an unobtrusive tool for long-term sleep studies. In ACM SIGHIT symposium on International health informatics - IHI '12, ACM (2012), 71-80.

3. Chen, L., Pan, G., and Li, S. Touch-driven interaction via an NFC-enabled smartphone. In PerCom '12 Workshops (2012), 504-506.

4. Cheng, J., Amft, O., and Lukowicz, P. Active capacitive sensing: Exploring a new wearable sensing modality for activity recognition. In Pervasive '10 (2010), 319-336.

5. Cohn, G., Morris, D., Patel, S., and Tan, D. Humantenna: using the body as an antenna for real-time whole-body interaction. In CHI '12 (2012), 1901-1910.

6. Cohn, G., Morris, D., Patel, S. N., and Tan, D. S. Your noise is my command: sensing gestures using the body as an antenna. In CHI 'll (2011), 791-800.

7. Crispin, X., Jakobsson, F. L. E., Crispin, A., Grim, P. C. M., Andersson, P., Volodin, A., Haesendonck, C. V., Auweraer, M. V. D., Salaneck, W. R., and Berggren, M. The origin of the high conductivity of (pedot-pss) plastic electrodes. Chemistry of Materials 18, 18 (2006), 4354-4360.

8. Dietz, P., and Leigh, D. Diamondtouch: A multi-user touch technology. In UIST '01 (2001), 219-226.

9. Dodson, B., and Lam, M. Micro-interactions with nfc-enabled mobile phones. In Mobile Computing, Applications, and Services, vol. 95. Springer, 2012, 118-136.

10. Fei, S., Webb, A. M., Kerne, A., Qu, Y., and Jain, A. Peripheral Array of Tangible NFC Tags: Positioning Portals for Embodied Trans-Surface Interaction. In ITS '13 (2013).

11. Foster, K. R., and Lukaski, H. C. Whole-body impedance-what does it measure? The American journal of clinical nutrition 64, 3 (1996), 388S-396S.

12. Goertzel, G. An algorithm for the evaluation of finite trigonometric series. American Mathematical Monthly 65, 1 (1958), 34-35.

13. Grosse-Puppendahl, T., Berghoefer, Y., Braun, A., Wimmer, R., and Kuijper, A. OpenCapSense: A Rapid Prototyping Toolkit for Pervasive Interaction using Capacitive Sensing. In PerCom '13 (2013), 152-159.

14. Grosse-Puppendahl, T., Braun, A., Kamieth, F., and Kuijper, A. Swiss-Cheese Extended: An Object Recognition Method for Ubiquitous Interfaces based on Capacitive Proximity Sensing. In CHI '13 (2013), 1401-1410.
15. Harrison, C., Sato, M., and Poupyrev, I. Capacitive fingerprinting: exploring user differentiation by sensing electrical properties of the human body. In UIST '12 (2012), 537-544.

16. Ishii, H., and Ullmer, B. Tangible Bits: Towards Seamless Interfaces between People, Bits and Atoms. In CHI '97, ACM (1997), 234-241.

17. Kawahara, Y., Hodges, S., Cook, B. S., Zhang, C., and Abowd, G. D. Instant inkjet circuits: Lab-based inkjet printing to support rapid prototyping of ubicomp devices. In UbiComp '13, ACM (2013), 363-372.

18. Liu, J., Johns, E., Atallah, L., Pettitt, C., Lo, B., Frost, G., and Yang, G.-Z. An intelligent food-intake monitoring system using wearable sensors. In $B S N$ ' 12 , IEEE (2012), 154-160.

19. McGookin, D., Robertson, E., and Brewster, S. Clutching at straws: using tangible interaction to provide non-visual access to graphs. In $\mathrm{CHI}$ ' 10 , ACM (2010), 1715-1724.

20. Murata Manufacturing Co, Ltd. Capacitive Coupling Power Transmission Module, 2011. http://www.murata.com/products/article/pdf/ta1291.pdf (accessed 2014-02-25).

21. NFC Forum. Protocol Technical Specification, 2014. http://members.nfc-forum.org/specs/spec_list/ (accessed 2014-02-25).

22. Paradiso, J., and Starner, T. Energy scavenging for mobile and wireless electronics. Pervasive Computing, IEEE (2005), 18-27.

23. Park, D. G., Kim, J. K., Sung, J. B., Hwang, J. H., Hyung, C. H., and Kang, S. W. TAP: touch-and-play. In CHI '06, ACM (2006), 677-680.

24. Riedenklau, E., Hermann, T., and Ritter, H. Tangible Active Objects and Interactive Sonification as a Scatter Plot Alternative for the Visually Impaired. In International Conference on Auditory Display, ACM (2010).

25. Sato, M., Poupyrev, I., and Harrison, C. Touché: enhancing touch interaction on humans, screens, liquids, and everyday objects. In CHI '12 (2012), 483-492.

26. Savage, V., Zhang, X., and Hartmann, B. Midas: Fabricating Custom Capacitive Touch Sensors to Prototype Interactive Objects. In UIST '12 (2012).

27. Schmidt, A., Shirazi, A., and van Laerhoven, K. Are you in bed with technology? Pervasive Computing, IEEE 11, 4 (Oct 2012), 4-7.

28. Smith, J. R. Electric Field Imaging. PhD thesis, Massachusetts Institute of Technology, 1999.

29. Sousa, M., Techmer, A., Steinhage, A., Lauterbach, C., and Lukowicz, P. Human tracking and identification using a sensitive floor and wearable accelerometers. In PerCom '13 (2013), 166-171. 
30. Stikic, M., Huynh, T., Van Laerhoven, K., and Schiele, B. ADL Recognition Based on the Combination of RFID and Accelerometer Sensing. In Pervasive Health 2008, IEEE (January 2008), 258-263.

31. STMicroelectronics Inc. STRFNFCA Near field communication transceiver, 2014. http://www.st.com/stweb-ui/static/active/en/resource/technical/document/ datasheet/DM00052610.pdf (accessed 2014-06-17).

32. Texas Instruments Inc. Application Report SLOA184 NFC and RFID Reader Ultra-Low-Power Card Presence Detection Using MSP430 and TRF79xxA, 2014. http://www.ti.com/lit/an/sloa184/sloa184.pdf (accessed 2014-02-25).

33. Texas Instruments Inc. TRF7970A Multiprotocol Fully Integrated 13.56-MHz RFID and Near Field Communication (NFC) Transceiver IC, 2014. http://www.ti.com/lit/ds/slos743k/slos743k.pdf (accessed 2014-02-25).

34. Valtonen, M., Mäentausta, J., and Vanhala, J. TileTrack : Capacitive Human Tracking Using Floor Tiles. In PerCom '09 (2009), 121-133.
35. Vu, T., and Gruteser, M. Personal Touch-Identification Tokens. IEEE Pervasive Computing 12, 2 (2013), 10-13.

36. Wimmer, R., and Baudisch, P. Modular and Deformable Touch-Sensitive Surfaces Based on Time Domain Reflectometry. In UIST'11, ACM (2011), 517-526.

37. Wimmer, R., Kranz, M., Boring, S., and Schmidt, A. A Capacitive Sensing Toolkit for Pervasive Activity Detection and Recognition. In PerCom '07 (2007), 171-180.

38. Yu, N.-H., Chan, L.-W., Lau, S. Y., Tsai, S.-S., Hsiao, I., Tsai, D.-J., Hsiao, F.-I., Cheng, L.-P., Chen, M., Huang, P., et al. TUIC: enabling tangible interaction on capacitive multi-touch displays. In $\mathrm{CHI}$ ' 11 , ACM (2011), 2995-3004.

39. Zimmerman, T. G. Personal Area Networks (PAN): Near-Field Intra-Body Communication. IBM Systems Journal 35, 3\&4 (2013), 609-617.

40. Zimmerman, T. G., Smith, J. R., Paradiso, J. A., Allport, D., and Gershenfeld, N. Applying electric field sensing to human-computer interfaces. In CHI '95 (1995), 280-287. 